\title{
$\beta$-Nicotinamide Mononucleotide, an Anti-Aging Candidate Compound, Is Retained in the Body for Longer than Nicotinamide in Rats
}

\author{
Tomoyo KaWAmura, Noriyuki Mori and Katsumi SHIBATA* \\ Department of Nutrition, School of Human Cultures, The University of Shiga Prefecture, \\ 2500 Hassakacho, Hikone, Shiga 522-8533, Japan \\ (Received February 18, 2016)
}

\begin{abstract}
Summary The turnover of the oxidized form of nicotinamide adenine dinucleotide $\left(\mathrm{NAD}^{+}\right)$has attracted interest in regard to longevity. Thus, compounds that can rapidly increase the cellular $\mathrm{NAD}^{+}$concentration have been surveyed by many researchers. Of those, $\beta$-nicotinamide mononucleotide ( $\beta$-NMN) has been focused on. Studies on the biosynthesis of $\mathrm{NAD}^{+}$from $\beta$-NMN have been reported at the cellular level, but not at the whole animal level. In the present study, we investigated whether $\beta$-NMN is superior to nicotinamide (Nam) as a precursor of $\mathrm{NAD}^{+}$in whole animal experiments. To this end we compared the $\mathrm{NAD}^{+}$concentration in the blood and the urinary excretion amounts of $\mathrm{NAD}^{+}$ catabolites. Rats were intraperitoneally injected with $\beta$-NMN or Nam. After the injection, blood samples and urine samples were collected at 3-h intervals. The concentration of blood total NAD $\left(\mathrm{NAD}^{+}+\mathrm{NADH}\right)$ in each sample showed no significant differences between the two groups. The urinary excretion amounts of $\mathrm{NAD}^{+}$catabolites in the urine samples collected at 3-6 $\mathrm{h}$ after the injection were lower in the $\beta$-NMN group than in the Nam group. These results suggest that $\beta$-NMN is retained in the body for longer than Nam.
\end{abstract}

Key Words $\beta$-nicotinamide mononucleotide, nicotinamide phosphoribosyltransferase, $\mathrm{NAD}^{+}$, urine, rat

More than 2,200 kinds of enzymes are known, and about 500 of them need the oxidized form of nicotinamide adenine dinucleotide $\mathrm{NAD}^{+}$, the oxidized form of nicotinamide adenine dinucleotide phosphate $\left(\mathrm{NADP}^{+}\right)$, and their reduced forms NADH and NADPH as coenzymes. Recently, it has been shown that the deacetylation reaction of the aging-related molecule, sirtuin, needs $\mathrm{NAD}^{+}$as a substrate (1). It has attracted attention as a link between metabolism and aging. The NAD ${ }^{+}$ biosynthesis pathway has been classified into a de novo pathway from tryptophan (Trp) and a salvage pathway from nicotinamide (Nam). In particular, the salvage pathway couples with the sirtuin deacetylation reaction, and reuses Nam from deacetylation, converting it into $\beta$-nicotinamide mononucleotide ( $\beta$-NMN) by nicotinamide phosphoribosyltransferase (Nampt). Moreover, nicotinamide mononucleotide adenylyltransferase (Nmnat) synthesizes $\mathrm{NAD}^{+}$from $\beta$-NMN and ATP. Nampt is the rate-limiting $\mathrm{NAD}^{+}$biosynthesis enzyme, and it plays a critical role in biological aspects (e.g., metabolism, inflammation and aging) by adjusting the $\mathrm{NAD}^{+}$level in response to nutrition and the environment (2). In addition, it has been reported that $\beta$-NMN improves age-associated metabolic diseases, such as diabetes and Alzheimer, because $\mathrm{NAD}^{+}$metabolites such as

\footnotetext{
*To whom correspondence should be addressed.

E-mail: kshibata@shc.usp.ac.jp
}

$\beta$-NMN increase the $\mathrm{NAD}^{+}$level (3-5). It has also been reported that after $\beta$-NMN treatment, the $\mathrm{NAD}^{+}$level in a 22-mo-old mouse is similar to that in a 6 -mo-old mouse (6). Hence, it is thought that $\beta$-NMN has antiaging effects.

It has been reported that the liver $\mathrm{NAD}^{+}$level in rat was significantly increased by injection of a large amount of Nam, which is an $\mathrm{NAD}^{+}$precursor (7). However, Nam is not converted into $\mathrm{NAD}^{+}$by the $\mathrm{Nam} \rightarrow \beta$ $\mathrm{NMN} \rightarrow \mathrm{NAD}^{+}$pathway. $\mathrm{NAD}^{+}$derived from Nam is made by the Nam $\rightarrow$ nicotinic acid $(\mathrm{NiA}) \rightarrow$ nicotinic acid mononucleotide $(\mathrm{NaMN}) \rightarrow$ nicotinic acid adenine dinucleotide $(\mathrm{NaAD}) \rightarrow \mathrm{NAD}^{+}$pathway, because Nampt is inhibited by NAD $(8,9)$. On the other hand, $\mathrm{NAD}^{+}$ synthesis from $\beta$-NMN is not regulated by the cellular $\mathrm{NAD}^{+}$level. In light of metabolic control mechanisms and many reports on $\beta$-NMN, $\beta$-NMN is likely to be more effective as an $\mathrm{NAD}^{+}$precursor than Nam. Studies on $\mathrm{NAD}^{+}$biosynthesis from $\beta$-NMN have been conducted at the cellular levels, but not at the whole animal level.

It has been previously shown that urinary excretion of water-soluble vitamins correlates with their intake (10). In addition, urine collection is easy and noninvasive (10). That is, the measurement of metabolite excretion in the urine is very useful.

In the present study, we investigated whether $\beta$-NMN is superior to Nam as a precursor of $\mathrm{NAD}^{+}$in whole animal experiments. 

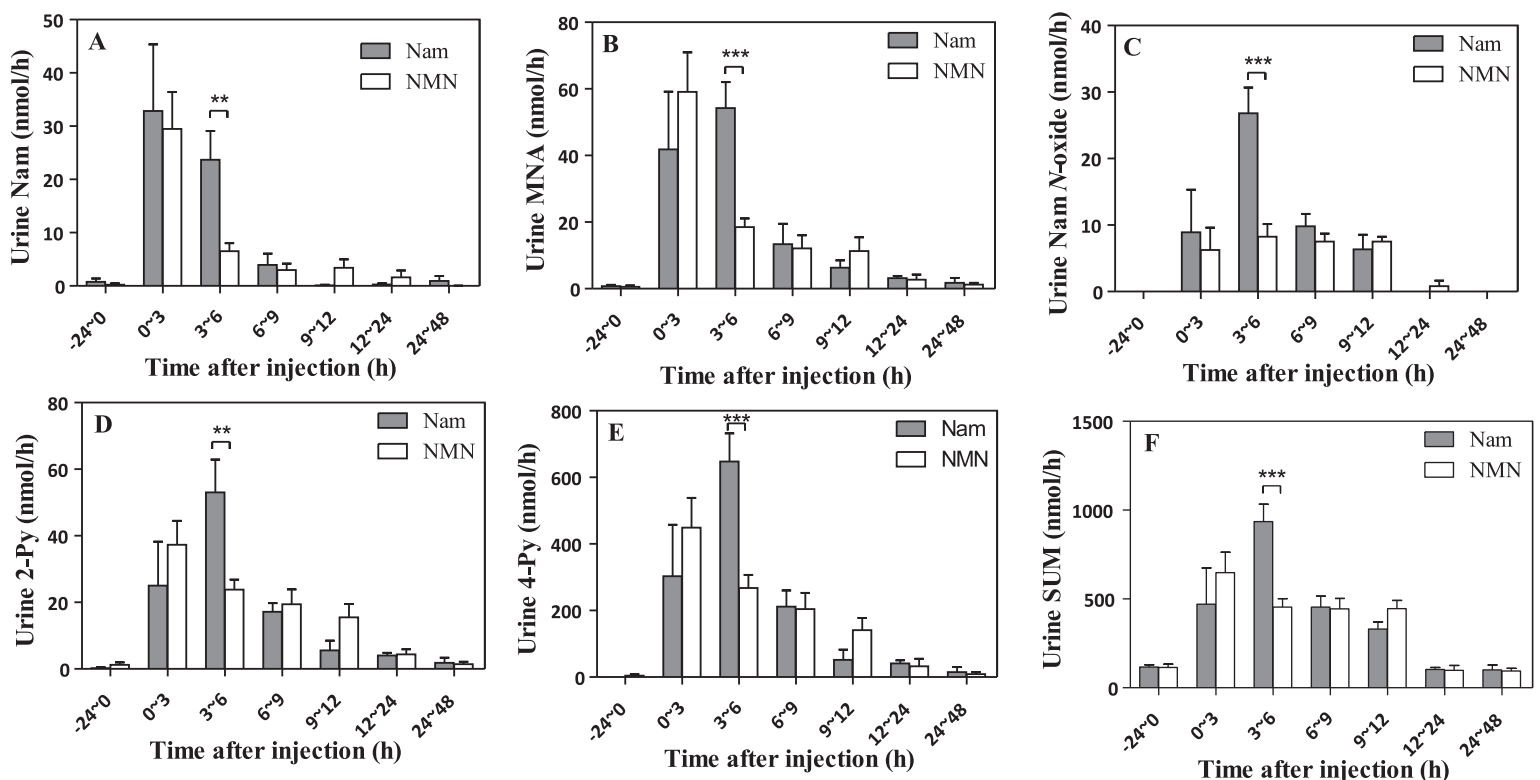

Fig. 1. The changes in $\mathrm{NAD}^{+}$metabolites excreted to the urine at $-24-0,0-3,3-6,6-9,9-12,12-24$ and $24-48 \mathrm{~h}$ after Nam or $\beta$-NMN was administered to rats. Forty-five micromoles of Nam or $\beta$-NMN per kg body weight were intraperitoneally injected into rats at $0 \mathrm{~h}$. Values are mean \pm SE, $n=5-6$. The values in a row without a common superscript letter differ, $p<0.05$, as determined by one-way ANOVA followed by Tukey's multiple comparison tests. (A) Urine Nam. (B) Urine MNA.

(C) Urine Nam N-oxide. (D) Urine 2-Py. (E) Urine 4-Py. (F) Urine SUM.

\section{Materials and Methods}

Reagents. Vitamin-free milk casein, sucrose and L-methionine were purchased from Wako Pure Chemical Industries, Ltd. (Osaka, Japan). Corn oil was purchased from Ajinomoto (Tokyo, Japan). Gelatinized cornstarch, a mineral mixture (AIN-93G mineral mixture) (11), a vitamin mixture, NiA-free AIN-93 vitamin mixture containing 25\% choline bitartrate (11), and $\beta$-NMN were obtained from Oriental Yeast Co., Ltd. (Tokyo, Japan).

Nam $\left(\mathrm{C}_{6} \mathrm{H}_{6} \mathrm{~N}_{2} \mathrm{O}, \mathrm{MW}=122.13\right)$ was purchased from Wako Pure Chemical Industries. $\mathrm{NAD}^{+}$was purchased from Sigma (St. Louis, MO). $N^{1}$-Methylnicotinamide (MNA) chloride $\left(\mathrm{C}_{7} \mathrm{H}_{9} \mathrm{~N}_{2} \mathrm{O}-\mathrm{HCl}, \quad \mathrm{MW}=159.61\right)$ was purchased from Tokyo Chemical Industry (Tokyo, Japan). $\quad N^{1}$-Methyl-2-pyridone-5-carboxamide (2-Py) $\left(\mathrm{C}_{7} \mathrm{H}_{8} \mathrm{~N}_{2} \mathrm{O}_{2}, \mathrm{MW}=152.15\right)$ and $\mathrm{N}^{1}$-methyl-4-pyridone3-carboxamide (4-Py) $\left(\mathrm{C}_{7} \mathrm{H}_{8} \mathrm{~N}_{2} \mathrm{O}_{2}, \mathrm{MW}=152.15\right)$ were synthesized by the methods of Pullman and Colowick (12), and Shibata et al. (13), respectively. All other chemicals used were of the highest purity available from commercial sources.

Animal treatment and diets.

Ethical approval of the study protocol: The care and treatment of experimental animals conformed to the guidelines set by the University of Shiga Prefecture (Shiga, Japan) for the ethical treatment of laboratory animals. The room was maintained at $\sim 22^{\circ} \mathrm{C}$ with $\sim 60 \%$ humidity. A 12 -h light-dark cycle (06:0018:00/18:00-06:00) was used.

Kinetics of the urinary excretion of $\mathrm{NAD}^{+}$catabolites after Nam or $\beta$-NMN administration. To acclimatize rats to their new environment, male Wistar rats (7 wk old; CLEA Japan, Inc., Tokyo) were kept in individual rat metabolic cages (CT-10; CLEA Japan). Rats were fed ad libitum a $20 \%$ casein diet consisting of $20 \%$ vitaminfree milk casein, $0.2 \%$ L-methionine, $46.9 \%$ gelatinizedcornstarch, $23.4 \%$ sucrose, $5 \%$ corn oil, 3.5\% AIN-93G mineral mixture and 1\% NiA-free AIN-93 vitamin mixture containing $25 \%$ choline bitartrate for $5 \mathrm{~d}$. The rats were then divided into three groups: on the 6 th day at 09:00 h, $45 \mu \mathrm{mol} / \mathrm{kg}$ Nam dissolved in $0.5 \mathrm{~mL}$ of sterilized saline was intraperitoneally injected into the rats of the first group; $45 \mu \mathrm{mol} / \mathrm{kg} \beta$-NMN dissolved in $0.5 \mathrm{~mL}$ of sterilized saline was injected into rats of the second group; and $0.5 \mathrm{~mL}$ of sterilized saline was injected into the rats of the third group (control). Urine samples were collected in amber bottles containing $1 \mathrm{~mL}$ of $1 \mathrm{~mol} / \mathrm{L}$ $\mathrm{HCl}$ at $-24-0 \mathrm{~h}, 0-3 \mathrm{~h}, 3-6 \mathrm{~h}, 6-9 \mathrm{~h}, 9-12 \mathrm{~h}, 12-24 \mathrm{~h}$ and $24-48 \mathrm{~h}$ after the injection and stored at $-25^{\circ} \mathrm{C}$ until use.

Kinetics of blood $\mathrm{NAD}^{+}$concentrations after Nam or NMN administration. To acclimatize rats to their new environment, male Wistar rats (7 wk old; CLEA Japan) were kept in cages and fed ad libitum the $20 \%$ casein diet described above for $5 \mathrm{~d}$. The rats were then divided into three groups: at 09:30 h on the 6th day, $45 \mu \mathrm{mol} /$ $\mathrm{kg}$ body weight (BW) Nam dissolved in $0.5 \mathrm{~mL}$ of sterilized saline was intraperitoneally injected into the rats of the first group; $45 \mu \mathrm{mol} / \mathrm{kg} \mathrm{BW} \beta$-NMN dissolved in $0.5 \mathrm{~mL}$ of sterilized saline was injected into the rats of the second group; and $0.5 \mathrm{~mL}$ of sterilized saline was injected into the rats of the third group (control). Blood samples were collected from the tail vein every $30 \mathrm{~min}$ for $6 \mathrm{~h}$ after the injection.

Comparison of the liver NAD concentrations, $3 \mathrm{~h}$ after 


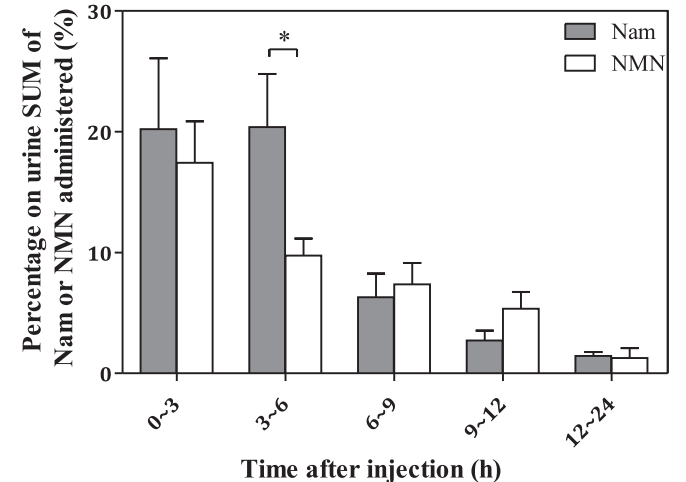

Fig. 2. Percentage of the urinary SUM of Nam or $\beta$-NMN administered. Forty-five micromoles of Nam or $\beta$-NMN per kg body weight were intraperitoneally injected into rats at $0 \mathrm{~h}$. Values are mean \pm SE, $n=5-6$. * Statistical difference from the Nam group, determined by Student's $t$-test.

Nam or $\beta$-NMN administration. To acclimatize rats to their new environment, male Wistar rats (12 wk old; CLEA Japan) were kept in cages and fed ad libitum the $20 \%$ casein diet described above for $5 \mathrm{~d}$. The rats were then divided into three groups: at 09:30 h on the 6th day, $45 \mu \mathrm{mol} / \mathrm{kg}$ BW Nam dissolved in $0.5 \mathrm{~mL}$ of sterilized saline was intraperitoneally injected into the rats of the first group; $45 \mu \mathrm{mol} / \mathrm{kg} \mathrm{BW} \beta$-NMN dissolved in $0.5 \mathrm{~mL}$ of sterilized saline was injected into the rats of the second group; and $0.5 \mathrm{~mL}$ of sterilized saline was injected into the rats of the third group (control). The rats were killed by decapitation at $3 \mathrm{~h}$ after the administration, the liver of each animal was dissected, and a portion $(\sim 0.25 \mathrm{~g})$ was immediately treated as described in the literature (14).

Measurement of $\mathrm{NAD}^{+}$catabolites. $\mathrm{NAD}^{+}$catabolites, such as Nam $(13,15)$, Nam N-oxide $(15,16)$, MNA (17), 2-Py $(13,15)$ and 4-Py $(13,15)$, in the urine were measured as described previously. The SUM was defined as the total amount of $\mathrm{Nam}+\mathrm{Nam}$ $\mathrm{N}$-oxide $+\mathrm{MNA}+2-\mathrm{Py}+4-\mathrm{Py}$.

Calculation method for the percentage of the urinary SUM of Nam or $\beta$-NMN administered. We assumed that the urinary SUM originating from food was constant during the experiment. Each percentage of the urinary SUM of Nam or $\beta$-NMN administered $(\%)=\{$ (urinary SUM of the Nam or $\beta$-NMN-administered group, mol/urine collected hours) - (urinary SUM of the control group, mol/ urine collected hours $)\} \div$ (Nam or $\beta$-NMN administered, $\mathrm{mol}) \times 100$. The urine collection hours were $0-3 \mathrm{~h}$, 3-6 h, 6-9 h, 9-12 h, and 12-24 h.

Measurement of total NAD in the blood and liver. Total $\mathrm{NAD}\left(\mathrm{NAD}^{+}+\mathrm{NADH}\right)$ in whole blood and liver was measured using the methods of Shibata and Murata (14).

Statistical analysis. Values are reported as mean \pm SE. Differences between the Nam group and the $\beta$-NMN group were analyzed by Student's $t$-test. $p<0.05$ was considered statistically significant. Prism version 5.0 was used for all analyses.

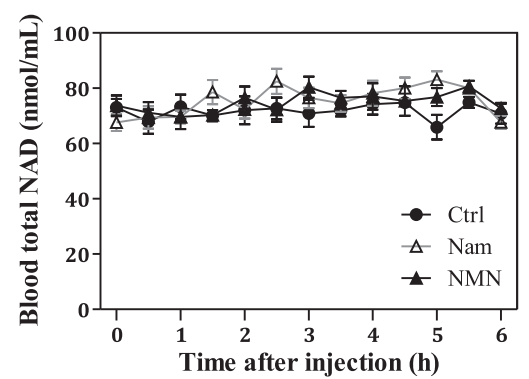

Fig. 3. The changes in blood total $\mathrm{NAD}\left(\mathrm{NAD}^{+}+\mathrm{NADH}\right)$ concentration at $0,0.5,1.0,1.5,2.0,2.5,3.0,3.5,4.0$, $4.5,5.0,5.5$ and $6.0 \mathrm{~h}$ after Nam or $\beta$-NMN administration into rats. Forty-five micromoles of Nam or $\beta$-NMN per kg body weight were intraperitoneally injected into rats at $0 \mathrm{~h}$. Values are mean $\pm \mathrm{SE}, n=6$. A significant difference was not observed, as determined by one-way ANOVA followed by Tukey's multiple comparison tests.

\section{Results}

Kinetics of the urinary excretion of $\mathrm{NAD}^{+}$catabolites after Nam or $\beta$-NMN administration

Figure 1 shows the changes in urinary excretion amounts of $\mathrm{NAD}^{+}$catabolites, such as Nam, Nam $N$-oxide, MNA, 2-Py and 4-Py, and their SUM (=Nam+ $\mathrm{Nam} N$-oxide $+\mathrm{MNA}+2-\mathrm{Py}+4$-Py) after Nam or $\beta$-NMN administration. Interestingly, the urinary excretion of all the tested catabolites was lower in the $\beta$-NMN group than in the Nam group at 3-6 h. Moreover, the percentage of the urinary SUM was lower in the $\beta$-NMN-administered group than in the Nam group at 3-6 h (Fig. 2). On the other hand, the percentages at 6-9 and 9-12 h tended to be higher in the $\beta$-NMN group than in the Nam group.

Kinetics of the blood total NAD $\left(\mathrm{NAD}^{+}+\mathrm{NADH}\right)$ concentration after Nam or $\beta$-NMN administration

The diets used in the present study did not contain niacin. Therefore, all total NAD was derived from dietary Trp and injected Nam or $\beta$-NMN. As shown in Fig. 3, the concentration of blood total NAD at each time point showed no significant differences among the three groups.

Comparison of the liver total NAD $\left(\mathrm{NAD}^{+}+\mathrm{NADH}\right)$ concentration 3 h after Nam or $\beta$-NMN administration

The concentration of liver total NAD at $3 \mathrm{~h}$ after Nam or $\beta$-NMN administration also showed no significant differences among the three groups (the values were around $800 \mathrm{nmol} / \mathrm{g}$ of liver).

\section{Discussion}

The cellular $\mathrm{NAD}^{+}$concentration is kept constant by the Nampt step, which catalyzes the reaction, Nam + 5-phosphoribosyl-1-pyrophosphate $(\mathrm{PRPP}) \rightarrow \beta$ $\mathrm{NMN}+$ inorganic pyrophosphate. $\mathrm{NAD}^{+}$synthesis from Nam in the liver proceeds only when the $\mathrm{NAD}^{+}$ level decreases below the physiological concentration, because Nampt is inhibited by $\mathrm{NAD}^{+}$(18). Consequently, even if Nam is injected, the $\mathrm{NAD}^{+}$level is hard to increase. However, the reaction that produces $\mathrm{NAD}^{+}$ 


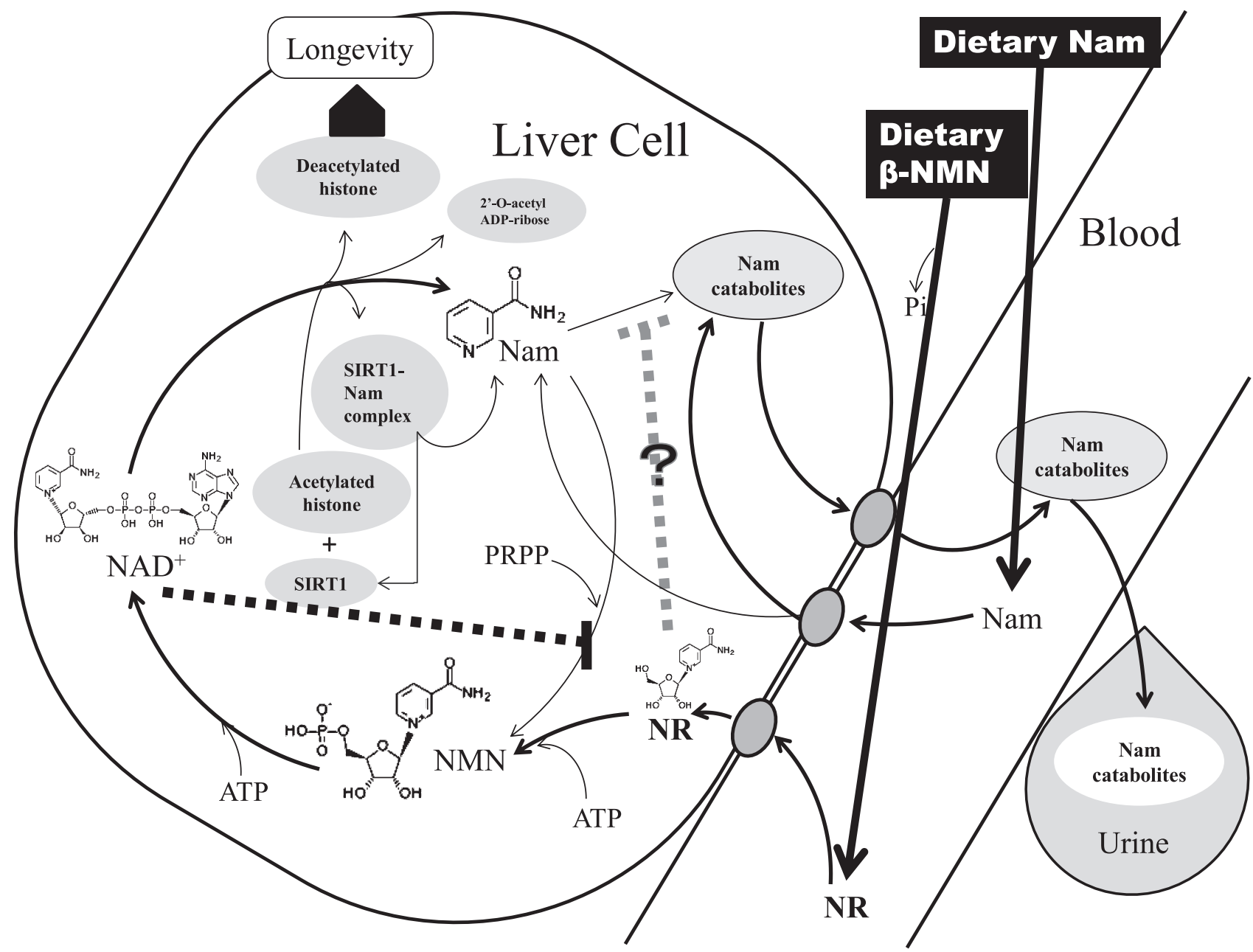

Fig. 4. Proposed $\mathrm{NAD}^{+}$biosynthesis pathways from Nam and $\beta$-NMN. Nam mainly catabolized to form inactive niacin compounds such as Nam N-oxide, MNA, 2-Py and 4-Py, which are eliminated into the urine. This is because the reaction, Nam + 5-phosphoribosyl-1-pyrophosphate (PRPP) $\rightarrow \beta$-NMN + pyrophosphate, which is catalyzed by Nam phosphoribosyltransferase (Nampt), is inhibited by the physiological concentration of $\mathrm{NAD}^{+}$. Therefore, administration of Nam does not contribute to the increased turnover of salvage biosynthesis of $\mathrm{NAD}^{+}$biosynthesis. If $\beta$-NMN is administered, it is dephosphorylated and is converted to nicotinamide riboside (NR) and it appears as NR in the bloodstream. NR is transported into liver cells and is re-phosphorylated to form $\beta$-NMN. This step (NR $+\mathrm{ATP} \rightarrow \beta$-NMN $+\mathrm{ADP}$ ) should be the rate-limiting step of the conversion of NR to NAD ${ }^{+}$. Thus, accumulation of NR should be observed in liver cells. The increased concentration of NR may inhibit the reaction of Nam $\rightarrow$ Nam catabolites. The resulting phenomenon accelerates the turnover of salvage biosynthesis of $\mathrm{NAD}^{+}$, which activates the SIRT1 reaction, because SIRT1 (histone deacetylase) needs NAD ${ }^{+}$. Deacetylated histone molecules induce DNA silencing, contributing to anti-aging and longevity.

from $\beta$-NMN is not controlled by the cellular $\mathrm{NAD}^{+}$ level; therefore when $\beta$-NMN is injected, the $\mathrm{NAD}^{+}$levels increase easily.

In the present study, we compared the blood total $\mathrm{NAD}\left(\mathrm{NAD}^{+}+\mathrm{NADH}\right)$ and urinary excretion amounts of $\mathrm{NAD}^{+}$catabolites in $\beta$-NMN- and Nam-administered rats. The concentration of blood total NAD and liver total NAD showed no significant differences among the three groups. However, when we examined the kinetics of the urinary excretion, the urinary excretion of the SUM was lower in the $\beta$-NMN group than in the Nam group at 3-6 $\mathrm{h}$ after the administration. Moreover, the percentage on the urinary SUM was much lower in the $\beta$-NMN group than in the Nam group at $3-6 \mathrm{~h}$. This result suggests that $\beta$-NMN is retained in the body for longer than Nam is. In addition, this result means that
$\beta$-NMN has a higher turnover of salvage biosynthesis of $\mathrm{NAD}^{+}$than Nam does. The resulting phenomenon accelerates the turnover of salvage biosynthesis of $\mathrm{NAD}^{+}$, which activates the SIRT1 reaction, because SIRT1 (histone deacetylase) needs $\mathrm{NAD}^{+}$. Deacetylated histone molecules induce DNA silencing, contributing to anti-aging and longevity.

The proposed biosynthesis pathway from Nam and $\beta$-NMN in liver cells is shown in Fig. 4 . When $\beta$-NMN is intraperitoneally injected, $\beta$-NMN is dephosphorylated in the blood to form Nam riboside (NR), which is then transported into the cells. NR is re-phosphorylated to form $\beta$-NMN, which is then synthesized to $\mathrm{NAD}^{+}$in the nucleus. In contrast to Nam, which is controlled at the Nam $\rightarrow \beta$-NMN reaction, the $\beta$-NMN biosynthesis pathway is not regulated. To silence DNA, activation 
of SIRT1 (histone deacetylase) is necessary (1). Thus, administration of $\beta$-NMN might be a good method for suppressing aging.

\section{Acknowledgments}

This study was part of a project entitled "Nutritional studies on B-group vitamins" (principal investigator: Katsumi Shibata), which was supported by Public Utility Foundation for the Vitamin \& Biofactor Society. This work was conducted at the Department of Nutrition, School of Human Cultures, The University of Shiga Prefecture.

\section{Authors' contributions}

TK, NM and KS designed the study. TK and KS drafted the manuscript. NM critically read the draft paper and gave valuable comments. TK and NM performed the experiments. All authors read and approved the final manuscript.

\section{REFERENCES}

1) Imai S, Armstrong CM, Kaeberlein M, Guarente L. 2000. Transcriptional silencing and longevity protein Sir2 is an NAD-dependent histone deacetylase. Nature 403: 795-800.

2) Imai S, Yoshino J. 2013. The importance of NAMPT/ NAD/SIRT1 in the systemic regulation of metabolism and ageing. Diabetes Obes Metab 3: 26-33.

3) Yoshino J, Mills KF, Yoon MJ, Imai S. 2011. Nicotinamide mononucleotide, a key $\mathrm{NAD}(+)$ intermediate, treats the pathophysiology of diet- and age-induced diabetes in mice. Cell Metab 14: 528-536.

4) Canto C, Houtkooper RH, Pirinen E, Youn DY, Oosterveer MH, Cen Y, Fernandez-Marcos PJ, Yamamoto H, Andreux PA, Cettour-Rose P, Gademann K, Rinsch C, Schoonjans K, Sauve AA, Auwerx J. 2012. The NAD(+) precursor nicotinamide riboside enhances oxidative metabolism and protects against high-fat diet-induced obesity. Cell Metab 15: 838-847.

5) Gong B, Pan Y, Vempati P, Zhao W, Knable L, Ho L, Wang J, Sastre M, Ono K, Sauve AA, Pasinetti GM. 2013. Nicotinamide riboside restores cognition through an upregulation of proliferator-activated receptor- $\gamma$ coactivator $1 \alpha$ regulated $\beta$-secretase 1 degradation and mitochondrial gene expression in Alzheimer's mouse models. Neurobiol Aging 34: 1581-1588.

6) Gomes AP, Price NL, Ling AJ, Moslehi JJ, Montgomery MK, Rajman L, White JP, Teodoro JS, Wrann CD, Hubbard BP, Mercken EM, Palmeira CM, de Cabo R, Rolo AP,
Turner N, Bell EL, Sinclair DA. 2013. Declining NAD(+) induces a pseudohypoxic state disrupting nuclearmitochondrial communication during aging. Cell 155: 1624-1638.

7) Kaplan NO, Goldin A, Humphreys SH, Ciotti MM, Stolzenbach FE. 1956. Pyridine nucleotide synthesis in the mouse. J Biol Chem 219: 287-298.

8) Ijichi H, Ichiyama A, Hayaishi O. 1966. Studies on the biosynthesis of nicotinamide adenine dinucleotide. 3. Comparative in vivo studies on nicotinic acid, nicotinamide, and quinolinic acid as precursors of nicotinamide adenine dinucleotide. J Biol Chem 241: 3701-3707.

9) Petrack P, Greengard P, Kalinsky H. 1966. On the relative efficacy of nicotinamide and nicotinic acid as precursors of nicotinamide adenine dinucleotide. J Biol Chem 241: 2367-2372.

10) Shibata K, Hirose J, Fukuwatari T. 2014. Relationship between urinary concentrations of nine water-soluble vitamins and their vitamin intakes in Japanese adult males. Nutr Metab Insights 7: 61-75.

11) Reeves RG. 1998. Components of the AIN-93 diets as improvements in the AIN-76A diet. J Nutr 127: 838S-841S.

12) Pullman ME, Colowick SP. 1954. Preparation of 2- and 6-pyridones of $\mathrm{N}^{1}$-methylnicotinamide. J Biol Chem 206: 121-127.

13) Shibata K, Kawada T, Iwai K. 1988. Simultaneous micro-determination of nicotinamide and its major metabolites, $N^{1}$-methyl-2-pyridone-5-carboxamide and $N^{1}$-methyl-3-pyridone-4-carboxamide, by high-performance liquid chromatography. J Chromatogr 424: 23-28.

14) Shibata K, Murata K. 1986. Blood NAD as an index of niacin nutrition. Nutr Int 2: 177-181.

15) Shibata K. 1989. High-performance liquid chromatographic measurement of nicotinamide $\mathrm{N}$-oxide in urine after extracting with chloroform. Agric Biol Chem 53: 1329-1331.

16) Maeta A, Sano M, Fukuwatari T, Shibata K. 2014. Simultaneous measurement of nicotinamide and its catabolites, nicotinamide $N$-oxide, $N^{1}$-methyl-2-pyridone-5-carboxamide, and $N^{1}$-methyl-4-pyridone-3-carboxamide, in mice urine. Biosci Biotechnol Biochem 78: 1306-1309.

17) Shibata K. 1987. Ultramicro-determination of $N^{1}$-methylNam in urine by high-performance liquid chromatography. Vitamins 61: 599-604 (in Japanese).

18) Shibata K, Hayakawa T, Taguchi H, Iwai K. 1991. Regulation of pyridine nucleotide coenzyme metabolism. Adv Exp Med Biol 294: 207-218. 